\title{
Influence of obstacles on bubbles rising in water-saturated sand
}

\author{
Raphaël Poryles ${ }^{1}$, Germán Varas ${ }^{2, \star}$, and Valérie Vidal ${ }^{1, \star \star}$ \\ ${ }^{1}$ Univ. Lyon, ENS de Lyon, Univ. Claude Bernard, CNRS, Laboratoire de Physique, F-69342 Lyon, France \\ ${ }^{2}$ Instituto de Física, Pontificia Universidad Católica de Valparaíso, Av. Universidad 330, Valparaíso, Chile
}

\begin{abstract}
This work investigates the dynamics of air rising through a water-saturated sand confined in a HeleShaw cell in which a circular obstacle is trapped. The air is injected at constant flow rate through a single nozzle at the bottom center of the cell. Without obstacle, in a similar configuration, previous studies pointed out the existence of a fluidized zone generated by the central upward gas motion which entrains two granular convection rolls on its sides. Here, a circular obstacle which diameter is of the order of the central air channel width is trapped at the vertical of the injection nozzle. We analyze the influence of the obstacle location on the size of the fluidized zone and its impact on the morphology of the central air channel. Finally, we quantify the variations of the granular free surface. Two configurations with multiple obstacles are also considered.
\end{abstract}

\section{Introduction}

Gas rising in liquid-saturated sands is a phenomenon widely encountered in nature, with strong implications in natural or industrial hazard mitigation. Examples of soil liquefaction, methane venting areas [1], $\mathrm{CO}_{2}$ sequestration [2] and air sparging for ground decontamination [3] are among the many which focus the attention of both fundamental and applied research since decades. Since the beginning of the 90's, the remediation of contaminated soils has motivated studies of the morphology of gas invasion in saturated granular media. In the particular case of a single gas injection point in highly packed beds, it has been shown that the gas rising through the immersed granular layer generates specific patterns. Cumulated in time, the gas pathways delimitate a zone of parabolic shape above the injection point [3-5]. For mobile sands (e.g. loosepacked sands), the gas and fluid recirculation generate a so-called fluidized zone, at the vertical of the injection point. Its shape is also parabolic and consists of a central air channel with two granular convection rolls on the side, while outside this region, the grains remain at rest [6-11]. Most of these studies have been performed with either model sands, consisting in spherical glass beads, or real sands sieved in order to get a peaked size distribution, and further assess the influence of the grain size on the process. However, soils are intrinsically heterogeneous, with the presence of layering or large heterogeneities.

Ground heterogeneities can have drastic consequences on the dynamics of the sand layer fluidization. Although the case of a stratified medium modeled with rectangular, horizontal obstacles has been investigated qualitatively for highly packed beds [4], the influence of heterogeneities

${ }_{\star}^{\star}$ e-mail: german.varas@pucv.cl

${ }^{\star}$ e-mail: valerie.vidal@ens-lyon.fr trapped into mobile sediments has received, to our knowledge, few or no attention. In this work, we propose to investigate experimentally the air-induced fluidization of a mobile, water-saturated granular layer with a single or multiple trapped obstacle(s). The quasi-2D setup makes it possible to visualize the fluidized zone and quantify the influence of obstacles on its morphology.

\section{Experimental setup}

The experimental setup is a vertical Hele-Shaw cell (glass plates $40 \times 30 \mathrm{~cm}$, gap $e=2 \mathrm{~mm}$ ) filled with dense particles immersed in water. The particles are polydisperse spherical glass beads (Sovitec) with a typical diameter $d=318 \pm 44 \mu \mathrm{m}$ and a roughly gaussian size distribution [11]. The obstacle consists of a cylindrical magnet (HKCM Engineering e.K. S15x02Ni-N35, $1.17 \mathrm{~T}$ ) of diameter $D=1.5 \mathrm{~cm}$ and thickness $2 \mathrm{~mm}$, equal to the cell gap to prevent air, liquid or grains to flow on its faces. It is inserted in the empty cell by means of another magnet (cylinder of diameter $5 \mathrm{~mm}$ and height $28 \mathrm{~mm}$, HKCM Engineering e.K., Z05x28Ep-N52, 1.43 T) located outside the glass plates. This stronger magnet ensures that the obstacle remains fixed in the cell through all the experiment. The obstacle is located at the vertical of the air injection nozzle, with its center at height $h$ (Fig. 1a, third panel). The cell is then filled with grains and water, following a prococol described in [12] which ensures reproducible initial conditions. The initial granular bed height is fixed at $h_{g} \simeq 20 \mathrm{~cm}$, and the water layer above the grains free surface is $h_{w} \simeq 2 \mathrm{~cm}$.

Eight experiments are performed with different obstacle location $h=[2.5,5,7.5,10,12.5,15] \mathrm{cm}$ (Fig. 1a). A reference experiment without obstacle is also performed, as well as two additional experiments where three identical 

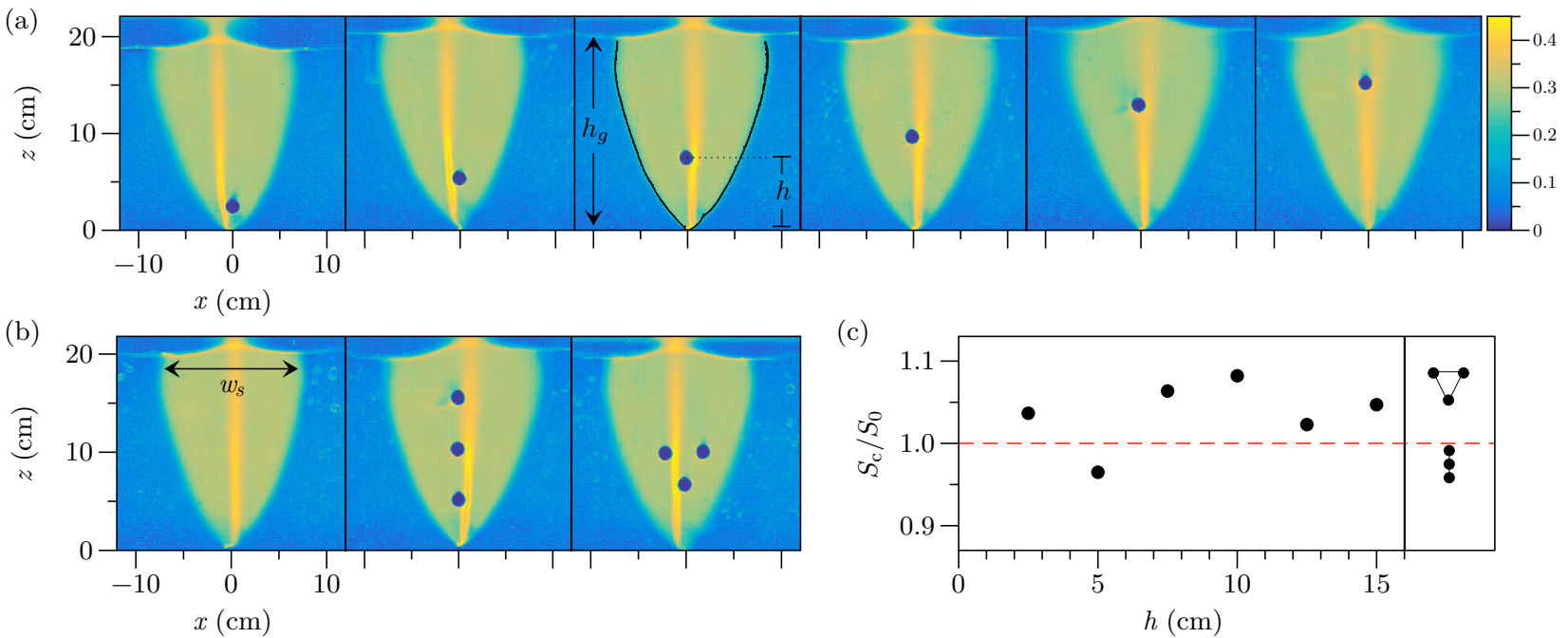

Figure 1. (a,b) Cumulated motion (matrix $M$, see text and Eq. 1) for the different experiments: (a) when varying the obstacle height $h$; (b) without obstacle (left), with three in-line obstacles (middle) and three obstacles with a triangular configuration (right). The colorbar indicates the percentage of motion in the system [threshold $1 \%$ ], $h_{g}$ the initial granular layer height, $h$ the obstacle height and $w_{s}$ the fluidized zone extension at $z=18 \mathrm{~cm}$. The black line (a, third image) denotes the contour of the fluidized zone (see text). (c) Surface of the zone delimited by this contour up to $z=18 \mathrm{~cm}, S_{\mathrm{c}}$ (see text), normalized by the surface of the fluidized zone without obstacle, $S_{0}$, as a function of the obstacle height [ $\bullet$ single obstacle (a); the line and triangle on the right of the figure correspond to the in-line (b, middle) or triangular (b, right) configuration]. The red dashed line indicates the reference without obstacle (Fig. 1b, left).

obstacles are set following two geometries (Fig. 1b): (1) the obstacles are located in-line, at the vertical of the injection point, at $h \simeq 5,10,15 \mathrm{~cm}$; (2) the obstacles are placed in a triangular shape, with a first obstacle at the vertical of the injection point at $h=7 \mathrm{~cm}$ and two more obstacles at $h=10.5 \mathrm{~cm}$, separated by a distance of $4 \mathrm{~cm}$.

At time $t=0 \mathrm{~s}$, air is injected from the bottom nozzle (inner diameter $1 \mathrm{~mm}$ ) at constant flow rate $Q=1.98 \mathrm{~mL} / \mathrm{s}$ (mass flow controller D-6311, M+W Instruments). The cell is lightened from behind by a LED panel. Images of the experiment are recorded with a camera (PixeLINK PL$\mathrm{B} 741 \mathrm{U}, 1280 \times 800 \mathrm{px}^{2}$ ) at a frame rate of 1 image every 2 seconds during 20 hours, which makes it possible to observe the long-time dynamics of the system.

\section{Results}

\subsection{Fluidized zone}

First we focus on the fluidized zone, which is the central region where the grains move during the experiment. To delimitate this region, we compute from the raw images $I_{k}$ a cumulated motion image $M$, defined as follows:

$$
M=\frac{1}{N} \sum_{k=1}^{N-1} \mathcal{B}_{1 \%}\left(\left|I_{k+1}-I_{k}\right|\right)
$$

where $N$ is the total number of frames, $\left|I_{k+1}-I_{k}\right|$ the absolute value of the difference between two consecutive images and $\mathcal{B}_{1 \%}$ a binarization function returning 1 when $\left|I_{k+1}-I_{k}\right|>1 \% \max \left(\left|I_{k+1}-I_{k}\right|\right)$, and 0 otherwise. The sum generates a matrix where each value corresponds to the number of images for which the corresponding pixel in the raw image sequence has changed its value by more than
1\%. After dividing by the total number of images (Eq. 1), $M$ thus represents the percentage of images - i.e., of time - a given spot has experienced motion (with a $1 \%$ threshold) during the whole experiment. Changing the threshold to 0.5 or $2 \%$ does not change significantly the results.

The images of cumulated motion for each experiment are displayed in Figures 1a and 1b. Without obstacle (Fig. 1b, left), we recover the parabolic shape reported in previous works [4-6, 8, 10, 13]. We compute the surface of the fluidized zone as the region where the percentage of cumulated motion is above $20 \%$ (third image, Fig. 1a). This threshold is chosen to match correctly the contour of the observed motion zone. As we are interested in the total area where the grains are affected both by the air rise and the presence of obstacles, we consider the zone defined by the fluidized zone contour including both the mobile region and the obstacle(s), up to $z=18 \mathrm{~cm}$ to avoid surface effects, and denote its surface $S_{\mathrm{c}}$. Figure 1c displays $S_{\mathrm{c}}$, normalized by the surface of the fluidized zone without obstacle, $S_{0}$, as a function of the obstacle height $h$. The case of multiple obstacles (in-line or triangular configuration) is also reported (Fig. 1c, right side). Except for $h=5 \mathrm{~cm}$ and the in-line configuration, where the height of the first obstacle is also $h=5 \mathrm{~cm}$, the presence of an obstacle tends to increase the size of the fluidized zone by a few percent.

\subsection{Central channel}

To investigate further the impact of the obstacle on the structure of the fluidized zone, we quantify in Figure 2 the bulk properties of the cumulated motion, and the average characteristics of the central channel. To do so, we consider the intensity profiles at constant height $z=z_{0}$ in the cumulated motion images, and fit for different $z_{0}$ the 

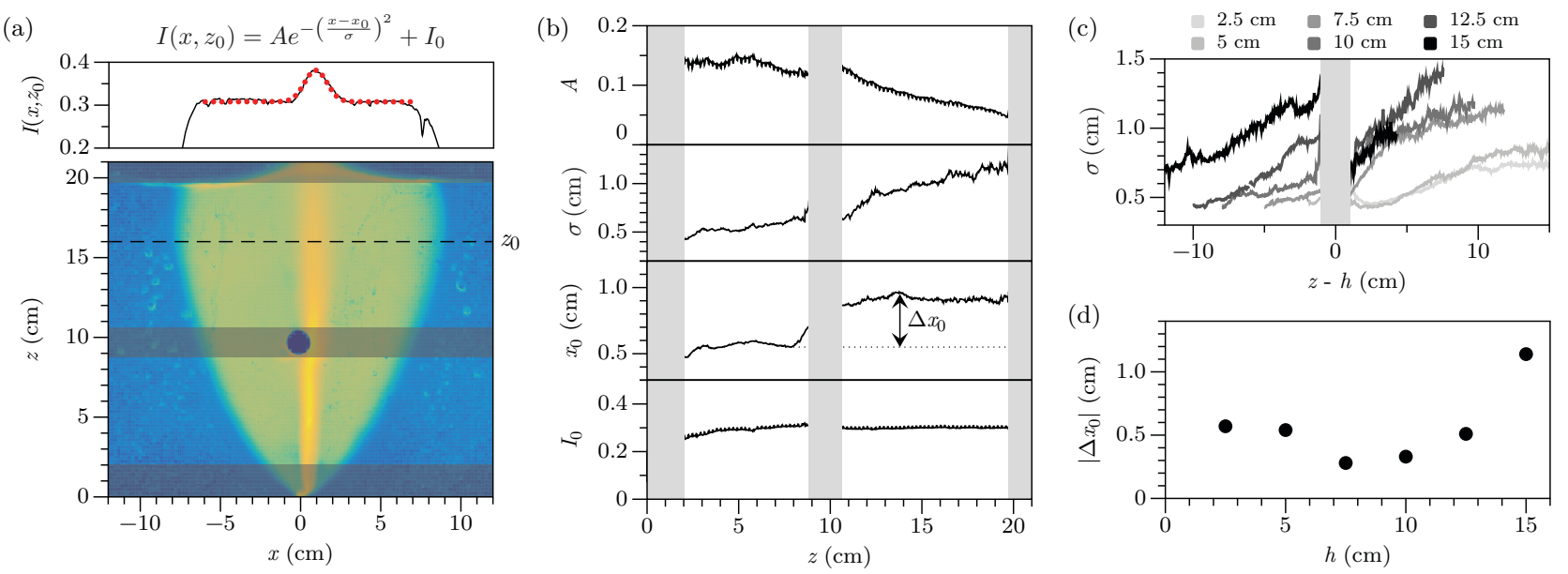

Figure 2. (a) Cumulated motion for $h=10 \mathrm{~cm}$ [bottom image]. The upper plot displays the intensity profile [solid black line] at $z=z_{0}$ [dashed line indicated in the image below], and the fit [red dots] corresponding to a gaussian function (the central channel) above a plateau (the granular convection). (b) Amplitude $A$, width $\sigma$, average horizontal shift $x_{0}$ and intensity plateau $I_{0}$ as a function of $z$ for the gaussian fit in (a). (c) Width $\sigma$ as a function of $z-h$ for different obstacle height $h$ [gray scale, see legend above]. (d) Horizontal shift $\left|\Delta x_{0}\right|$ of the central air channel due to the obstacle, as a function of the obstacle height $h$. The gray zones in (a,b,c) correspond to regions close to the injection nozzle, the obstacle and the free surface, which are ignored in the analysis.

granular convection and the central channel by a plateau and a gaussian function (Fig. 2a, upper panel, red dots):

$$
I\left(x, z_{0}\right)=A e^{-\left(\frac{x-x_{0}}{\sigma}\right)^{2}}+I_{0}
$$

The amplitude $A$ and width $\sigma$ can be interpreted as the average amplitude of motion and channel width, respectively. $A$ decreases while $\sigma$ increases along the channel height $z$ (Fig. 2b), which can be interpreted as the result of flow conservation in the central channel. The intensity plateau $I_{0}$ corresponding to the granular convection on the sides remains roughly constant (Fig. 2b), indicating that the presence of the obstacle, in average, has no influence on the side regions. Finally, note that the horizontal shift $x_{0}$ experiences a jump $\left|\Delta x_{0}\right|$ when passing the obstacle. In the gray zones (Figs. 2a,b,c) the gaussian fit does not work, due either to the proximity of the injection nozzle (the air channel is pinched and the distribution strongly peaked), the free surface disturbances or the obstacle.

Figures 2c,d summarize the experimental results with a single obstacle. The higher the obstacle, the larger is the width of the central channel both before $(z-h<0)$ and after $(z-h>0)$ the obstacle (Fig. 2c). The lateral jump $\left|\Delta x_{0}\right|$ experiences a slight decrease, then a noticeable increase for $h=7.5 \mathrm{~cm}$. Note that $\left|\Delta x_{0}\right|$ always remains smaller than the obstacle diameter, $D=1.5 \mathrm{~cm}$, although for $h=15 \mathrm{~cm}$, it reaches a value close to $D$.

\subsection{Grains free surface}

Lastly, we focus on the grains free surface behavior. Indeed, potential field applications are mostly based on surface observations, and the goal here is to find a possible link between the presence of obstacles and the surface morphology. To do so, we compute the mean free surface geometry by averaging in time the contour of the granular bed surface extracted from the raw images by simple contour detection. Figure 3 a compiles the average free surface vertical displacement, $\left\langle\Delta z_{s}\right\rangle$, as a function of the horizontal coordinate $x$, for different obstacle height. The red curve is the free surface deformation without obstacle, and $x=0 \mathrm{~cm}$ corresponds to the injection nozzle location. Note that even without obstacle, the maximum surface vertical displacement can exhibit a shift respect to the air injection point, here of about $1 \mathrm{~cm}$.

The first quantitative feature which can be extracted is the free surface extend, $w_{s}$. Because the initial height of the granular bed can slightly vary from one experiment to the other, and due to free surface fluctuations which may be difficult to quantify, we chose to estimate $w_{s}$ as the fluidized zone extent at $z=18 \mathrm{~cm}$, right below the free surface (Fig. 1b, left). Figure 3b displays $w_{s}$, normalized by the extent without obstacle, $w_{s}^{0}$. Interestingly, whichever the depth of the obstacle trapped in the granular bed, the extent of the fluidized zone at the surface is larger by a few percent $\left(w_{s} / w_{s}^{0}>1\right)$. A noticeable jump is observed for $h \geq 5 \mathrm{~cm}$, followed by a plateau, although no explanation can be given at present. To estimate further the free surface deformation, Fig. 3c displays the skewness $\gamma$ of the profiles shown in Fig. 3a. The skewness is a measurement of the profile asymmetry, defined as

$$
\gamma=\frac{\frac{1}{n} \sum_{i=1}^{n}\left(x_{i}-\bar{x}\right)^{3}}{\left(\sqrt{\frac{1}{n} \sum_{i=1}^{n}\left(x_{i}-\bar{x}\right)^{2}}\right)^{3}}
$$

where $\left\{x_{i}\right\}$ correspond to the $n^{\text {th }}$ discretized values of $\left\langle\Delta z_{s}\right\rangle$ and $\bar{x}$ to its average. A minimum is reported for $h=$ $7.5 \mathrm{~cm}$, at the same value where the plateau in $w_{s}$ appears.

The right part of the figure presents the in-line and triangular configurations for the three-obstacle configuration. Interestingly, the triangular geometry is close to the case without obstacle, while the in-line geometry is close to the single obstacle for $h=10 \mathrm{~cm}$. 

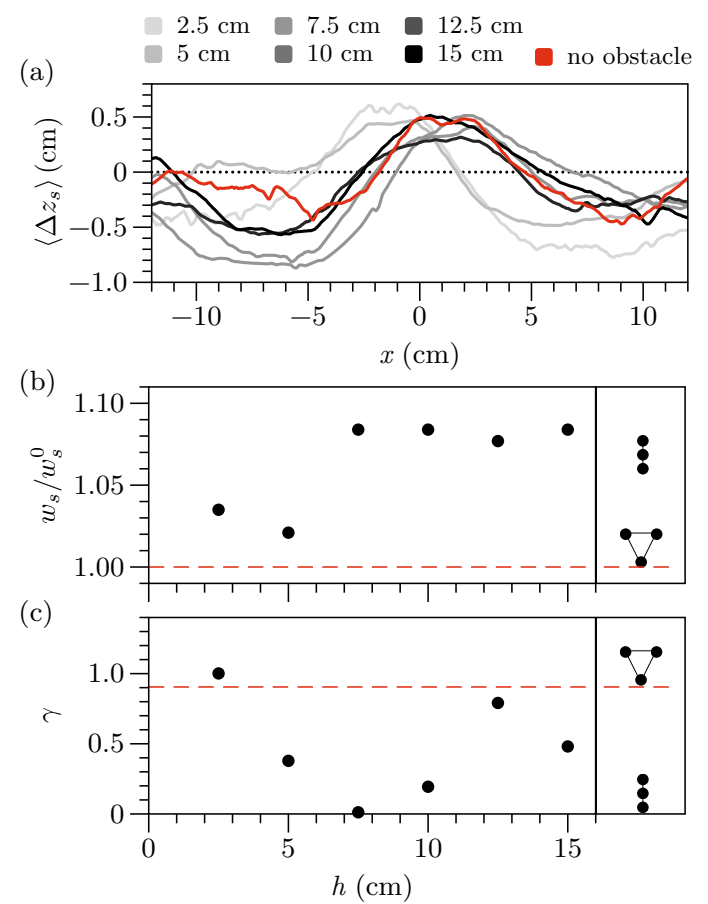

Figure 3. (a) Average vertical displacement of the grains free surface, $\left\langle\Delta z_{s}\right\rangle$, as a function of the horizontal coordinate, $x$ [the gray scale indicates the obstacle height and the red curve the experiment without obstacle, see legend above]. (b) Width of the fluidized zone measured at $z=18 \mathrm{~cm}, w_{s}$, normalized by the same width without obstacle, $w_{s}^{0}$, as a function of the obstacle height $h$. (c) Skewness $\gamma$ of the average free surface deformation displayed in (a) as a function of the obstacle height $h$. The red line in $(b, c)$ indicates the reference experiment without obstacle; the line and triangle symbols on the right correspond to the inline (Fig. 1b, middle) or triangular (Fig. 1b, right) configuration].

\section{Conclusion and perspectives}

We quantified the influence of obstacles on air rising in water-saturated sand. The presence of a single obstacle increases both the size of the central fluidized zone and its extent close to the surface, independently of its depth as long as the obstacle is not too close from the air injection point $(h \geq 7.5 \mathrm{~cm})$. The analysis of the average free surface deformation shows that its skewness undergoes a minimum at a critical height $h=7.5 \mathrm{~cm}$. No explication has been found at present for this peculiar value, although it could correspond to a transition between fingering and fracturing reported in a previous work [14]. A minimum is also observed at the same value for the horizontal shift in the central channel provoked by the obstacle.

In the field, obstacles would correspond to heterogeneities such as large grains or rocks trapped in sediments layer. This work demonstrates that even in a wellcontrolled experiment, the observation of the free surface is not sufficient to infer the depth of a ground heterogeneity. One can expect that the obstacle size or shape will play an important role on the surface deformation. It will be the central point of a further study which may unravel the origin of the critical height. The case of multiple obstacles is more complex, but closer to what is found in nature. Preliminary experiments considering two configurations with multiple obstacles show a discrepancy between the influence of the three obstacles on the fluidized zone size and its extent. A thorough study with multiple obstacles of different sizes, shapes and configurations is also among the perspectives of this work.

\section{Acknowledgements}

The authors acknowledge financial support from FONDECYT Project No. 11121300 and Programa de Cooperación Científica ECOS/CONICYT C14E07.

\section{References}

[1] H. Svensen, S. Planke, A.M.S. Renssen, B. Jamtveit, R. Myklebust, T.R. Eidem, S.S. Rey, Nature 429, 542 (2004)

[2] Q. Kang, I.N. Tsimpanogiannis, D. Zhang, P.C. Lichtner, Fuel Process. Technol. 86, 1647 (2005)

[3] K.R. Reddy, S. Kosgi, J. Zhou, Hazard. Waste Hazard. Mater. 12, 97 (1995)

[4] W. Ji, A. Dahmani, D.P. Ahlfeld, J.D. Lin, E. Hill, Ground Water Monit. Rem. 13, 115 (1993)

[5] J.S. Selker, M. Niemet, N.G. McDuffie, S.M. Gorelick, J.Y. Parlange, Transp. Porous Med. 68, 107 (2007)

[6] X.Z. Kong, W. Kinzelbach, F. Stauffer, Chem. Eng. Sci. 64, 1528 (2009)

[7] X.Z. Kong, W. Kinzelbach, F. Stauffer, Chem. Eng. Sci. 65, 4652 (2010)

[8] G. Varas, V. Vidal, J.C. Géminard, Phys. Rev. E 83, 011302 (2011)

[9] G. Varas, J.C. Géminard, V. Vidal, Gran. Matt. 15, 801 (2013)

[10] G. Varas, G. Ramos, J.C. Géminard, V. Vidal, Front. Phys. 3, 44 (2015)

[11] G. Ramos, G. Varas, J.C. Géminard, V. Vidal, Phys. Rev. E 92, 062210 (2015)

[12] R. Poryles, V. Vidal, G. Varas, Phys. Rev. E 93, 032904 (2016)

[13] R. Semer, J.A. Adams, K.R. Reddy, Geotech. Geol. Eng. 16, 59 (1998)

[14] V. De Zotti, V. Vidal, in Compte-rendus de la $17^{e}$ Rencontre du Non-Linéaire, edited by E. Falcon, M. Lefranc, F. Pétrélis, C.T. Pham (Non-Linéaire Publications, 2014), pp. 29-34 